\title{
Remarkable crystallization morphologies of poly(4-vinylpyridine) on single-walled carbon nanotubes in $\mathrm{CO}_{2}$-expanded liquids
}

\author{
M. Su ${ }^{1}$, Y. N. Wei ${ }^{2}$, L. Y. $Q i^{1}$, Y. C. Pang ${ }^{1}$, Y. Z. Guo ${ }^{1}, P . L i^{1}, J . L i^{1}, H . P . L i^{1 *}$ \\ ${ }^{1}$ Department of Chemistry, Zhengzhou University, Zhengzhou, Henan 450001, China \\ ${ }^{2}$ Physical Engineering College, Zhengzhou University, Henan 450001, China
}

Received 19 April 2011; accepted in revised form 4 July 2011

\begin{abstract}
Poly(4-vinylpyridine) (P4VP) is a widely studied polymer for applications in catalysis, humidity sensitive and antimicrobial materials due to its pyridine group exhibiting coordinative reactivity with transition metals. In this work, the non-covalent functionalization of single-walled carbon nanotubes (SWCNTs) with P4VP in $\mathrm{CO}_{2}$-expanded liquids (CXLs) is reported. It is found that P4VP stabilized SWCNTs show good dispersion in both organic solvent and aqueous solution $(\mathrm{pH}=2)$. The ability to manipulate the dispersion state of CNTs in water with P4VP will likely benefit many biological applications, such as drug delivery and optical sensors. Furthermore, the structure and morphology of P4VP/SWCNTs composite are examined, with the focus on molecular weight of P4VP (MW-P4VP), the pressure of CXLs and the concentration of P4VP. It is amazing that the $\mathrm{P}_{\mathrm{VP}} \mathrm{VP}_{1570}$ wrapping patterns undergo a notable morphological evolution from dotlike crystals to bottle brush-like, then to compact kebab-like, and then to widely-spaced dotted kebab patterns by facile pressure tuning in the higher polymer concentration series. In other words, the CXLs method enables superior control of the P4VP crystallization patterns on SWCNTs. Meanwhile, the CXL-assisted P4VP crystal growth mechanism on SWCNT is investigated, and the dominating growth mechanism is attributed to 'size dependent soft epitaxy' in $\mathrm{P}_{4} \mathrm{VP}_{15470} / \mathrm{SWCNTs}$ composites. We believe these studies would reveal great potential for P4VP in building up functional structures in CXLs that are responsive to environmental stimuli.
\end{abstract}

Keywords: polymer composites, single-walled carbon nanotubes, $\mathrm{CO}_{2}$-expanded liquids, non-covalent functionalization, poly(4-vinylpyridine)

\section{Introduction}

Polymer/CNT nanocomposites (PCN) belong to the most promising fields for carbon nanotubes (CNTs) $[1,2]$. The potential applications of PCNs include conductivity enhancement, electrostatic dissipation and aerospace structural materials, and a variety of polymers have been studied to form nanocomposites with CNTs depending on the targeted properties [3]. Meanwhile, wrapping CNTs with crystalline polymers is regarded as an ideal functionalization method considering the excellent mechanical properties of crystalline polymers as well as the poten- tial specific chain registry of different polymers upon CNTs. This method can retain the structural integrity of CNTs and simultaneously overcome the disability of the non-covalent modification method [4]. Poly(4-vinylpyridine) is chosen because it is a widely studied polymer for applications in catalysis [5-8], humidity sensitive [9] and antimicrobial materials [10] due to its pyridine group exhibting coordinative reactivity with transition metals. Being a hydrophobic polymer in apolar solvents and a cation polyelectrolyte in water at low $\mathrm{pH}[11,12]$, the $\mathrm{pH}-$ sensitive P4VP can be readily quaternized by alky-

\footnotetext{
${ }^{*}$ Corresponding author, e-mail: lihongping@zzu.edu.cn
} (c) BME-PT 
logen and then form positively charged polyelectrolytes as sensors and actuators [13, 14]. Thus, the $\mathrm{P} 4 \mathrm{VP} / \mathrm{CNT}$ nanocomposite is expected to play a vital role in exploring and developing the potential applications of the corresponding PCNs. In this work, the non-covalent attempts to wrapping crystalline P4VP on single-walled carbon nanotubes surface using $\mathrm{CO}_{2}$-expanded liquids (CXLs) method is introduced. It should be mentioned that CXLs are the most commonly used class of gas expanded liquids (GXLs) due to the safety and economic advantages of $\mathrm{CO}_{2}$. The ability of GXLs to readily pressure tune the mole fraction of the gaseous component, and thereby alter the solvating power, fluidity, and many other properties of these solvents proves advantageous in materials processing $[15,16]$, analytical separations [17] and organic reactions [1820] presents a great advantage. Polymer processing in CXLs taking advantage of the melting-point lowering and viscosity-lowering effects has been used to adjust particle size and morphology of polymers, and to facilitate foaming, impregnation, and comolding of polymers [21]. Moreover, GXLs/CXLs combine the beneficial properties of compressed gases and of conventional solvents, leading to a new class of tunable solvents that are often the ideal type of solvents for a given application while simultaneously reducing the environmental burden through substantial replacement of organic solvents with environmentally benign $\mathrm{CO}_{2}$ [21]. The challenging task is to achieve the controlled functionalization of SWCNTs by regulating the P4VP crystallization patterns. Accordingly, the influence of molecular weight of P4VP (MW-P4VP), polymer concentration, and the pressure of CXLs on the morphology change are examined in this work. Meanwhile, the mechanism of CXLs-assisted P4VP crystal growth on SWCNT is investigated. We believe that this work reports a new wrapping approach in CXLs to noncovalent engineering of SWCNTs surfaces, which leads to an enhancement of solubility of SWCNTs and enables superior control of the rela- tive placement of functionalities on the nanotube surface. Moreover, the material processing in CXLs also benefits from the milder operating conditions in contrast with the well-known supercrital antisolvent (SAS) processes [22].

\section{Experimental section}

\subsection{Materials}

SWCNTs (purity $>90 \mathrm{wt} \%$, outside diameter (OD) 1-2 nm, inside diameter (ID) $0.8-1.6 \mathrm{~nm}$, length 5$30 \mu \mathrm{m}$ ) were from Chengdu Organic Chemicals Co. Ltd., Chinese Academy of Sciences (Chengdu, China). Dimethyl sulphoxide (DMSO) and acetic anhydride $\left(\mathrm{CH}_{3} \mathrm{CO}\right)_{2} \mathrm{O}$ were supplied by the Damao Chemical Reagent Co., Ltd. (Tianjin, China). The monomer 4-vinyl pyridine (4VP) and Benzoyl Peroxide (BPO) were from Alfa Aesar (Beijing, China), and 2,2,6,6,-tetramethylpiperidine-N-oxyl (TEMPO) was supplied by Dengfeng Chemical Reagent Co., Ltd. (Tianjin, China). $\mathrm{CO}_{2}$ with a purity of $99.95 \%$ was provided by Zhengzhou Shuangyang Gas Co. (Zhengzhou, China). 4VP was purified prior to use by fractionated distillation. BPO was purified by reprecipitation of a saturated dried chloroform solution by methanol. TEMPO and $\left(\mathrm{CH}_{3} \mathrm{CO}\right)_{2} \mathrm{O}$ were used as received. $\mathrm{P}_{4 \mathrm{VP}_{9615}}\left(M_{\mathrm{n}}=9615 \mathrm{~g} \cdot \mathrm{mol}^{-1}\right)$ and $\mathrm{P}_{4 \mathrm{VP}_{15470}}\left(M_{\mathrm{n}}=15470 \mathrm{~g} \cdot \mathrm{mol}^{-1}\right)$ used in this work were prepared in our lab. SWCNTs were dried in a vacuum oven at $308.2 \mathrm{~K}$ for $24 \mathrm{~h}$ before use.

\subsection{Preparation of $P 4 V P$}

$\mathrm{P} \mathrm{VP}_{9615}$ was prepared by a method described by Fischer et al. [23], and $\mathrm{P} \mathrm{VP}_{15470}$ prepared according to the literature [24]. In a typical polymerization procedure, a two-necked round-bottom flask was loaded with an appropriate amount of TEMPO, $4 \mathrm{VP}, \mathrm{BPO}$, and/or $\left(\mathrm{CH}_{3} \mathrm{CO}\right)_{2} \mathrm{O}$; degassed by an argon gas purge; and sealed off under vacuum. The temperature of the polymerization and the duration was varied. P4VP formed, end-capped with TEMPO, was precipitated in hexane, and dried under vacuum. The detailed treatment conditions

Table 1. Polymerization of 4-vinyl pyridine at different reaction conditions

\begin{tabular}{|c|c|c|c|c|c|c|}
\hline Sample & $\begin{array}{c}\text { Time } \\
{[\mathbf{h}]}\end{array}$ & Accelerator & $\begin{array}{c}\text { Temperature } \\
{[\mathbf{K}]}\end{array}$ & $\begin{array}{c}\text { TEMPO/BPO } \\
\text { molar ratio }\end{array}$ & $\mathbf{M}_{\mathbf{n}}{ }^{\mathbf{a}}$ & $\mathbf{M}_{\mathbf{w}} / \mathbf{M}_{\mathbf{n}}{ }^{\mathbf{a}}$ \\
\hline 1 & 10.5 & - & $398.2-408.2$ & $0.65: 1$ & 9615 & 1.76 \\
\hline 2 & 16.0 & $\left(\mathrm{CH}_{3} \mathrm{CO}\right)_{2} \mathrm{O}^{\mathrm{b}}$ & $368.2-408.2$ & $1.30: 1$ & 15470 & 1.71 \\
\hline
\end{tabular}

${ }^{a}$ Number-average molecular weight $\left(M_{\mathrm{n}}\right)$ and polydispersity index $\left(M_{\mathrm{w}} / M_{\mathrm{n}}\right)$ were determined by Gel Permeation Chromatography (GPC) in DMF at $313.2 \mathrm{~K}$, flow rate $1 \mathrm{ml} / \mathrm{min}$

${ }^{\mathrm{b}} 1 \%(\mathrm{w} / \mathrm{w})$ 
for the preparation of P4VP, the number-average molecular weight $\left(M_{\mathrm{n}}\right)$ and polydispersity index $\left(M_{\mathrm{w}} / M_{\mathrm{n}}\right)$ of P4VP determined by Gel Permeation Chromatography (GPC) are provided in Table 1.

\subsection{The functionalization of SWCNTs by P4VP}

The experimental apparatus was described in our previous work [22]. Typically, appropriate amount of P4VP were dissolved in DMSO at a suitable temperature. $0.1 \mathrm{mg}$ of SWCNTs was dispersed in DMSO solution, the mixture was then ultrasonicated for $2-4 \mathrm{~h}$ at the temperature between $298.2-$ $318.2 \mathrm{~K}$ in order to disperse the SWCNTs homogeneously in the solution. The dispersion was then added into P4VP/DMSO solution in a tube. After equilibration, the tube containing the mixture was quickly transferred into a stainless-steel autoclave at the temperature of $338.2 \mathrm{~K} . \mathrm{CO}_{2}$ was then charged into the autoclave to achieve the desired pressure within a short time. After $3 \mathrm{~h}$ treatment in $\mathrm{CO}_{2}$ expanded DMSO, the system was slowly depressurized and the sample was collected and labeled. Comparative experiments of $\mathrm{P}_{4} \mathrm{VP}_{9615}$ or $\mathrm{P}_{4} \mathrm{VP}_{15470}$ decorated SWCNTs under the same experimental conditions free of $\mathrm{CO}_{2}$ were also performed.

To understand the polymer concentration dependence on CNTs modification, the reaction system are examined with the mass ratio of P4VP to SWCNTs selected to be 3:1 (i.e., $0.006 \mathrm{wt} \%$ P4VP to $0.002 \mathrm{wt} \%$ SWCNTs, the low polymer concentration series) and 5:1 (i.e., $0.010 \mathrm{wt} \% \mathrm{P} 4 \mathrm{VP}$ to $0.002 \mathrm{wt} \% \mathrm{SWC}$ NTs, the higher polymer concentration series), respectively.

\subsection{Characterization methods}

The number-average molecular weight $\left(M_{\mathrm{n}}\right)$ and polydispersity index $\left(M_{\mathrm{w}} / M_{\mathrm{n}}\right)$ of P4VP were determined by GPC (Waters 515 HPLC pump, Waters 2414 Refractive index detector; Waters Corporation, Milford, MA, USA), and are tabulated in Table 1. The morphology of P4VP functionalized SWCNTs was characterized by transmission electron microscopy (TEM). Samples were collected on a TEM grid and conducted using a JEOL JEM-2100 microscope with an accelerating voltage of $200 \mathrm{kV}$ (JEOL Ltd, Tokyo, Japan).

\section{Results and discussion}

\subsection{The enhancement of SWCNTs dispersibility using the CXLs method}

Figure 1 shows the organic and aqueous suspensions of SWCNTs stabilized with and without P4VP, here the modified SWCNTs are prepared at the reaction conditions of $\mathrm{P} 4 \mathrm{VP}_{15470}(0.05 \mathrm{wt} \%)$ and SWCNTs $(0.01 \mathrm{wt} \%)$ in $\mathrm{CO}_{2}$-DMSO at $8.30 \mathrm{MPa}$ $\left(x_{\mathrm{CO}_{2}}=0.425\right)$ and $338.2 \mathrm{~K}$ for $3 \mathrm{~h}$. It is shown that P4VP stabilized suspensions are homogeneous in either DMSO (Figure 1c) or aqueous hydrochloric acid solution ( $\mathrm{pH}=2$, Figure 1d) with no perceptible precipitation $24 \mathrm{~h}$ after ultrasonication. As expected, the suspensions of SWCNTs are close to complete sedimentation in DMSO (Figure 1a) or aqueous hydrochloric acid solution (Figure $1 \mathrm{~b}$ ) after $24 \mathrm{~h}$. The superior stability of suspensions stabilized with P4VP is attributed to increased non-covalent interactions between nanotubes and the P4VP. This results in an adsorbed layer of P4VP on the CNTs sidewalls, which brings about steric hindrance, preventing re-aggregation and subsequent precipitation of CNTs. We believe that the conformation of the adsorbed P4VP on CNTs would determine the dispersive behavior of as-prepared composites and their potential applications in many field. And what are the challenging factors in CXLs method to control the conformational transitions of P4VP wrap-

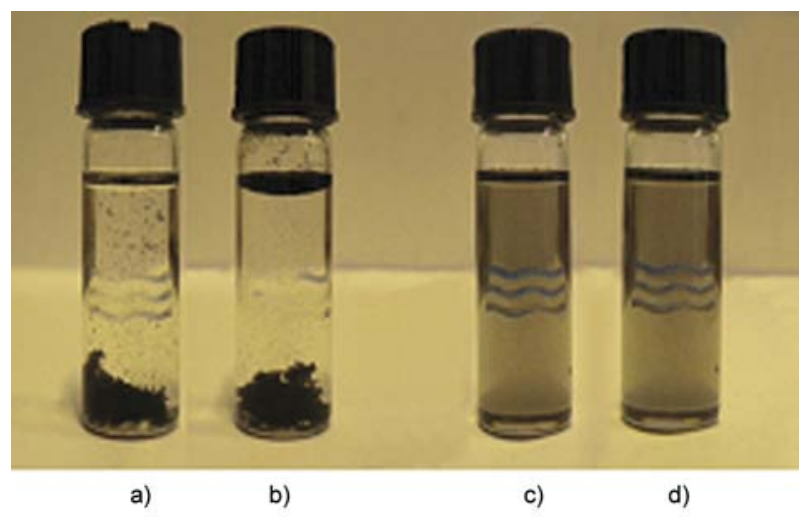

Figure 1. Organic and aqueous suspensions containing $0.010 \mathrm{wt} \%$ SWCNTs $24 \mathrm{~h}$ after ultrasonication (a) in DMSO; (b) in aqueous hydrochloric acid solution $(\mathrm{pH}=2)$; (c) the modified SWCNTs in DMSO; (d) the modified SWCNTs in aqueous hydrochloric acid solution $(\mathrm{pH}=2)$. The modified SWCNTs were prepared at the reaction conditions of $\mathrm{P} \mathrm{VP}_{15470}(0.050 \mathrm{wt} \%)$ and SWCNTs $(0.010 \mathrm{wt} \%)$ in DMSO at $8.30 \mathrm{MPa}\left(x_{\mathrm{CO}_{2}}=\right.$ 0.425 ) and $338.2 \mathrm{~K}$ for $3 \mathrm{~h}$. 
ping styles on CNTs? Accordingly, MW-P4VP, the mass ratio of $\mathrm{P} 4 \mathrm{VP}$ to $\mathrm{SWCNT}$, and the pressure of CXLs on the morphology change are examined.

\subsection{Effect of CXLs pressure and P4VP concentration on the modification of SWCNTs with P4VP $\mathbf{1 5 4 7 0}_{\text {- remarkable }}$ pressure dependence of morphological evolution}

In this section, the pressure and polymer concentration effect on the CNTs modification with $\mathrm{P} \mathrm{VP}_{15470}$ are examined with the mass ratio of P4VP to SWCNT selected to be 3:1 and 5:1, respectively. First of all, in an effort to investigate the pressure dependence, the reaction system are examined with the mass ratio of P4VP to SWCNTs as $3: 1$, and the pressures are chosen to be $7.99 \mathrm{MPa}\left(x_{\mathrm{CO}_{2}}=0.409\right)$, $9.29 \mathrm{MPa}\left(x_{\mathrm{CO}_{2}}=0.476\right), 11.32 \mathrm{MPa}\left(x_{\mathrm{CO}_{2}}=0.587\right)$ and $12.00 \mathrm{MPa}\left(x_{\mathrm{CO}_{2}}=0.626\right)$, respectively, along the bubble-point curve at $338.2 \mathrm{~K}_{\text {in }} \mathrm{CO}_{2}$-expanded DMSO [25]. The corresponding structures of P4VP $_{15470}$ decorated SWCNTs are shown in the TEM image (Figure 2a-2e). It is found that multiple P4VP periodic structures are formed with increasing pressure, i.e., the P4VP decoration on SWCNTs undergoes a wrapping pattern transition first from dots (Figure 2a) to compact kebabs (Figure 2b), then to less compact kebabs (Figure 2c) and next to sparsely dotted kebabs (Figure 2d-2e) as the pressure increases from 7.99 to $9.29,11.32$ and then to 12.00 MPa, respectively. A nanohybrid shish-kebab (NHSK) like structure is observed from Figure $2 b-$ 2e: the central shish is SWCNTs, with kebab-like P4VP crystals being regarded as periodically perpendicular to the CNTs axis. Typical NHSK structures have been reported previously $[4,26,27]$. The variation trend for the size and periodicity of the kebabs is indicated in Table 2: it is found that the periodicity of the kebab increases with increasing pressure whereas the size of the kebab is less pressure dependent.

The images from Figure 2a (7.99 MPa) indicate that at relatively low pressure, P4VP starts heterogeneous crystallization on SWCNTs as dot-like crystals. The mechanism is suggested as follows. In CXLs, $\mathrm{CO}_{2}$ cannot dissolve P4VP but is miscible with DMSO at suitable conditions, so $\mathrm{CO}_{2}$ is used as the antisolvent for P4VP/DMSO system. The volume of the DMSO-rich phase is expanded owing to the dissolution of $\mathrm{CO}_{2}$ and the solvent power on $\mathrm{P} 4 \mathrm{VP}$ is getting poorer, therefore P4VP molecules precipitate out of the supersaturated solution and start to be adsorbed on the surface of SWCNTs. So at relatively low pressure as $7.99 \mathrm{MPa}, \mathrm{P} 4 \mathrm{VP}$ molecules with folded-chains begin to align along SWCNTs as dots crystal so as to decrease the polymer surface energy, and consequently the CNTs surface is covered with small size of heteronucleus P4VP dots everywhere. The small dots crystal is formed due to the limited supply of P4VP in the vicinity of the CNTs during the crystal growth under lower $\mathrm{CO}_{2}$ solubility, and they could be regarded as a steady intermediate state, which could further form ordered patterns of nanohybrid structure under higher pressure. Generally, the solvent strength of the CXLs would further decrease due to more $\mathrm{CO}_{2}$ dissolved in DMSO, and therefore more P4VP can be deposited with increasing pressure. And what is the upcoming pressure dependent polymer crystal growth mechanism? We know that two possible factors could affect the nanohybrid conformation of P4VP crystal growth: the epitaxial growth of P4VP on CNTs and geometric confinement [3]. Here, the size of pristine SWCNTs (OD 1-2 nm) is smaller than that of $\mathrm{P} \mathrm{VP}_{15470}\left(R_{\mathrm{g}}\right.$, radius of gyration, about $10 \mathrm{~nm}$ [28] ). As a polymer with such a size diffuses

Table 2. Size and periodicity of the kebabs in the $\mathrm{P} 4 \mathrm{VP}_{15470} / \mathrm{SWCNTs}$ NHSK-like stucture produced in $\mathrm{CO}_{2}$-expanded DMSO at $338.2 \mathrm{~K}$

\begin{tabular}{|c|c|c|c|c|c|}
\hline Sample & $\begin{array}{l}\text { Pressure } \\
\text { [MPa] }\end{array}$ & $\mathbf{x}_{\mathrm{CO}_{2}}$ & $\begin{array}{l}\text { Lateral size of the kebabs } \\
\qquad[\mathrm{nm}]\end{array}$ & $\begin{array}{l}\text { Thickness of the kebabs } \\
\text { [nm] }\end{array}$ & $\begin{array}{l}\text { Periodicity of the kebabs } \\
{[\mathrm{nm}]^{\mathrm{a}}}\end{array}$ \\
\hline \multicolumn{6}{|c|}{ Reaction mixture with P4VP $15470(0.006 \mathrm{wt} \%)$ and SWCNTs $(0.002 \mathrm{wt} \%)$} \\
\hline $2 b$ & 9.29 & 0.476 & $35-40$ & 35 & - \\
\hline $2 c$ & 11.32 & 0.587 & $35-40$ & $25-35$ & $25-40$ \\
\hline $2 d$ & 12.00 & 0.626 & $30-35$ & $25-35$ & $40-75$ \\
\hline \multicolumn{6}{|c|}{ Reaction mixture with $\mathrm{P} \mathrm{VP}_{15470}(0.010 \mathrm{wt} \%)$ and SWCNTs $(0.002 \mathrm{wt} \%)$} \\
\hline $3 b$ & 8.30 & 0.425 & 25 & - & - \\
\hline $3 \mathrm{c}$ & 8.63 & 0.442 & $20-40$ & $10-25$ & - \\
\hline $3 d$ & 10.53 & 0.543 & $40-60$ & $40-60$ & $50-80$ \\
\hline
\end{tabular}

aPeriodicity is defined as the distance between the adjacent kekab centers 


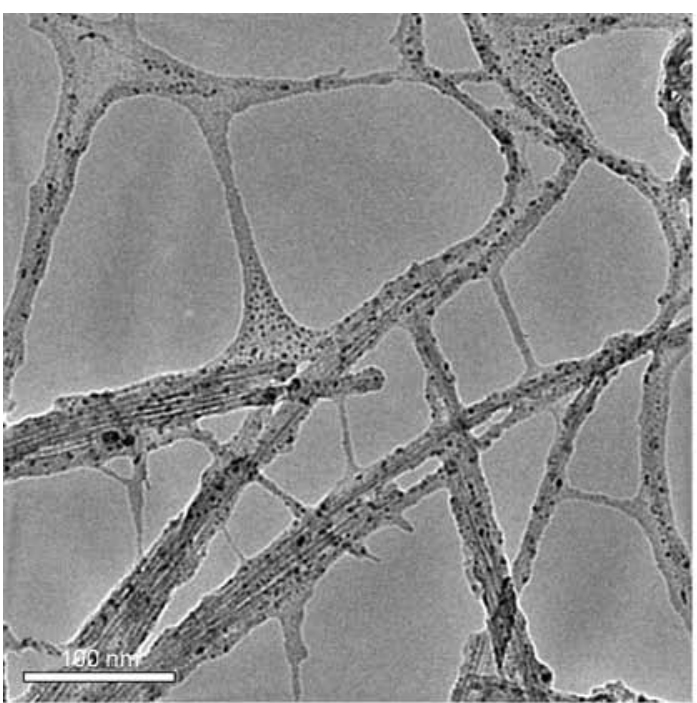

a)

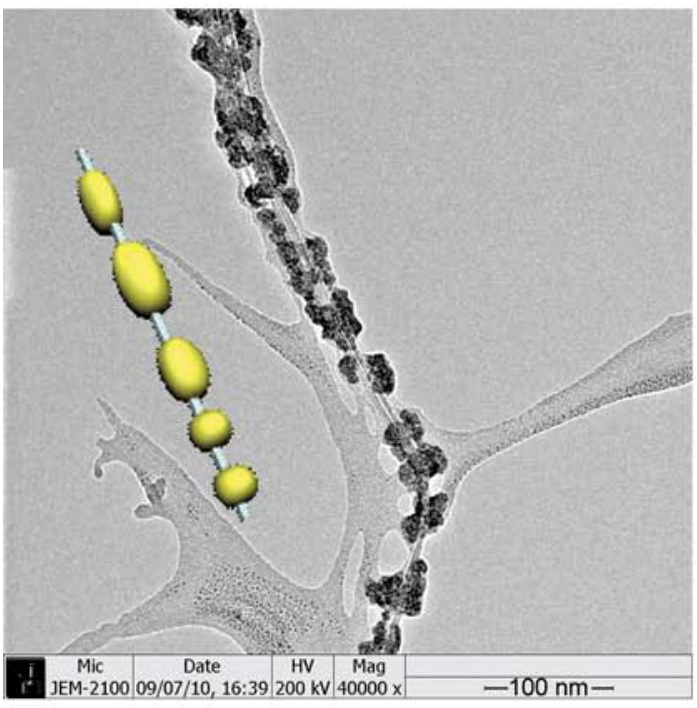

c)

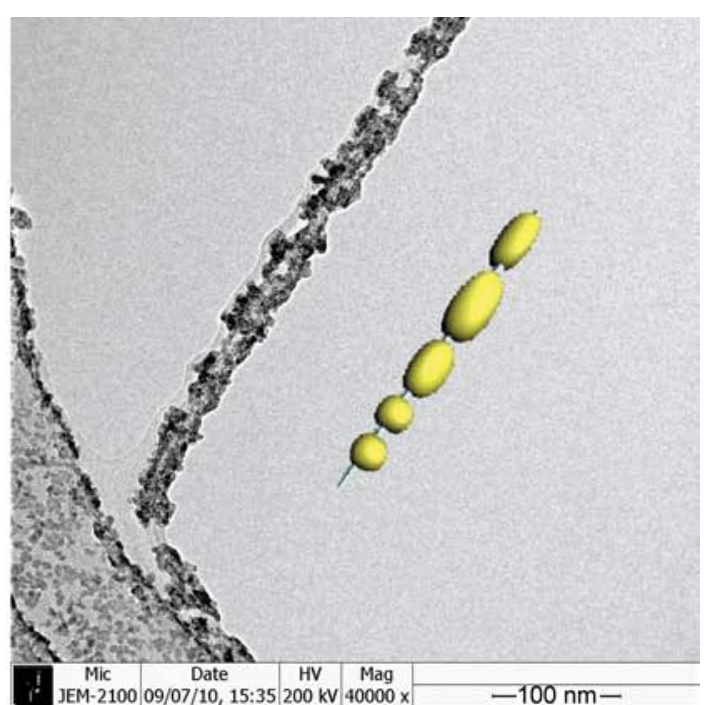

b)

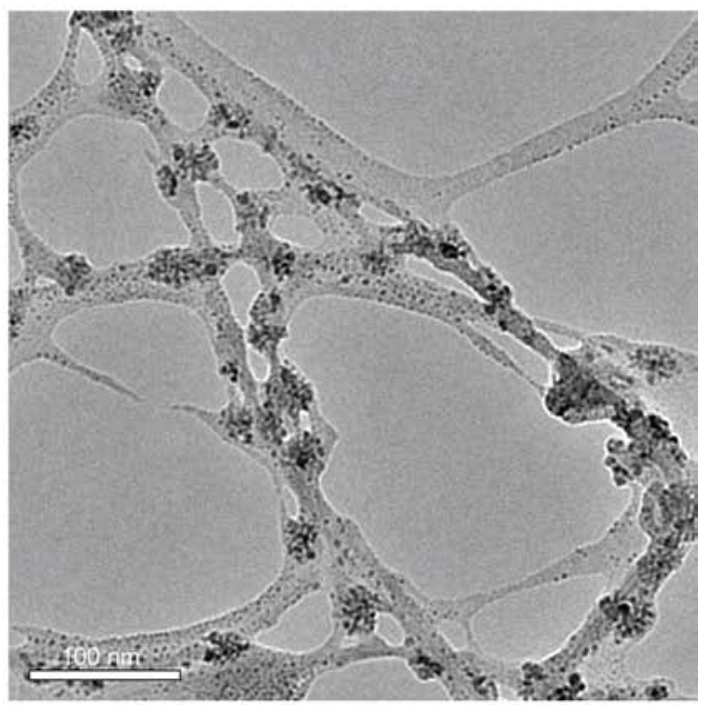

d)

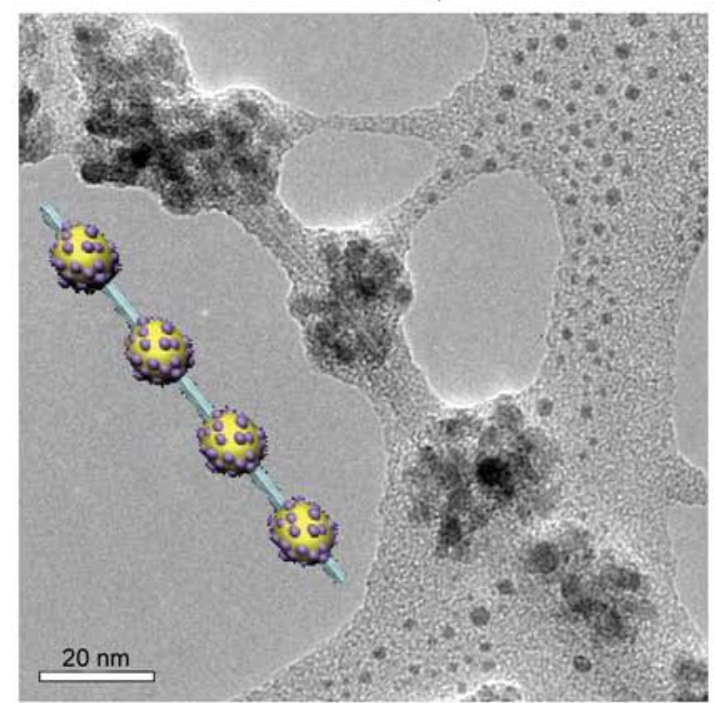

e)

Figure 2. TEM images of $\mathrm{P} 4 \mathrm{VP}_{15470}$-decorated SWCNTs obtained in $\mathrm{CO}_{2}$-expanded DMSO (338.2 K/varied pressure for $3 \mathrm{~h}$ ), with $0.006 \mathrm{wt} \% \mathrm{P}_{4} \mathrm{VP}_{15470}$ and $0.002 \mathrm{wt} \%$ SWCNTs in DMSO: (a) $7.99 \mathrm{MPa}\left(x_{\mathrm{CO}_{2}}=0.409\right)$; (b) $9.29 \mathrm{MPa}$ $\left(x_{\mathrm{CO}_{2}}=0.476\right)$; (c) $11.32 \mathrm{MPa}\left(x_{\mathrm{CO}_{2}}=0.587\right) ;(\mathrm{d}) /(\mathrm{e}) 12.00 \mathrm{MPa}\left(x_{\mathrm{CO}_{2}}=0.626\right)$. The schematic representations of the NHSK-like structure of the P4VP/SWCNTs composite are shown on image (b), (c) and (e), respectively. $\mathrm{P}_{4} \mathrm{VP}_{15470}$ form kebab-like crystals on SWCNTs surface with polymer chains parallel to the SWCNT axis. 
to the SWCNT surface and crystallizes, the diameter of the SWCNT plays a critical role in the formation of the crystal. Because of their small diameters, SWCNTs themselves can be considered as rigid macromolecules. Therefore, as $\mathrm{P} \mathrm{VP}_{15470}$ starts to crystallize onto this surface with further pressure increasing, geometric confinement is the major factor, and thus polymer chains are exclusively parallel to the CNT axis disregarding the CNT chirality. Consequently, the polymer crystal kebabs should be perpendicular to the CNT axis, and then a NHSK-like conformation is formed in $\mathrm{P} \mathrm{VP}_{15470} / \mathrm{SWCNTs}$ composite owing to the dominance of the 'size-dependent soft epitaxy' or geometric confinement factor [3]. Further morphological evolution with CXLspressure from compact kebabs (Figure 2b, $9.29 \mathrm{MPa}$ ) till dotted kebabs (Figure 2d-2e, $12.00 \mathrm{MPa}$ ) may be explained as follows. The precipitated P4VP can crystallize in two forms: (1) the homogeneous nucleation of P4VP itself and (2) the heterogeneous nucleation of P4VP on SWCNTs. Although the viscosity and surface tension of the CXLs will decrease with more $\mathrm{CO}_{2}$ dissolution in DMSO, which favors the adsorption of $\mathrm{P} 4 \mathrm{VP}$ on CNTs, the heteronucleus P4VP on CNTs may become less prevailing with increasing pressure whereas the homogeneous P4VP nucleation may consume the excessive increase in P4VP concentration. There may exist an threshold pressure around $10.00 \mathrm{MPa}\left(x_{\mathrm{CO}_{2}}=0.514\right)$ in our CXLs system, and the number of heterogeneous nucleation is supposed to be predominating over the homogeneous one below the threshold pressure, whereas the homogeneous nucleation is advantageous or preferential above the threshold pressure. The observation of compact kebabs at $9.29 \mathrm{MPa}$ (Figure 1b) conforms to the predominance of heteronucleus P4VP on CNTs at a pressure below the threshold pressure. The consequent less compact kebabs at 11.32 MPa (Figure 1c, periodicity 25$40 \mathrm{~nm}$ ) and the sparsely distributed dotted kebabs (Figure 2d-2e, periodicity $40-75 \mathrm{~nm}$ ) at $12.00 \mathrm{MPa}$ can be attributed to the preference of the competitive homogeneous nucleation of P4VP above the threshold pressure. And the higher the pressure above the threshold value, the less the number of heteronucleus $\mathrm{P} 4 \mathrm{VP}$ on CNT. In brief, this pressure tuning of competition or preference between homogeneous and heterogeneous nucleation would impede both the size and number increase of the "hybrid kebabs' with increasing pressure, which supports the observed structural transition in this low polymer concentration series. Therefore, we can conclude that CXLs pressure offers superior control over the period and structure of polymer crystallization patterns on SWCNTs.

In an effort to better understand the polymer concentration dependence, the modifications are performed in a higher polymer concentration series with the mass ratio of $\mathrm{P} 4 \mathrm{VP}_{15470}$ to SWCNTs as $5: 1$, and the pressures are chosen to be $7.79 \mathrm{MPa}$ $\left(x_{\mathrm{CO}_{2}}=0.399\right), 8.30 \mathrm{MPa}\left(x_{\mathrm{CO}_{2}}=0.425\right), 8.63 \mathrm{MPa}$ $\left(x_{\mathrm{CO}_{2}}=0.442\right)$ and $10.53 \mathrm{MPa}\left(x_{\mathrm{CO}_{2}}=0.543\right)$, respectively, along the bubble-point curve at $338.2 \mathrm{~K}$ in $\mathrm{CO}_{2}$-expanded DMSO [25]. The corresponding $\mathrm{P} 4 \mathrm{VP}$ conformations in $\mathrm{P}_{4} \mathrm{VP}_{15470}$-SWCNTs composites are reflected in Figure 3a-3d. Under higher mass ratio as 5:1, the corresponding morphology evolve from dots (Figure 3a) into bottle brush (Figure $3 b$ ), then to compact kebabs (Figure $3 c$ ), and next into dotted kebabs (Figure $3 \mathrm{~d}$ ) as the pressure increases from 7.79 to $8.30,8.63$ and then to $10.53 \mathrm{MPa}$, respectively. Surprisingly, an exceptional bottle-brush pattern of P4VP is observed at 8.30 MPa (Figure 3b). Herein, if the bottle-brush shaped $\mathrm{P} 4 \mathrm{VP}_{15470} / \mathrm{SWCNTs}$ composites could be imagined as a type of NHSK structure, with the SWCNTs the shish, and the ultrathin P4VP lamellae perpendicular to the CNTs axis, then a NHSK-like structure is again found for the higher polymer concentration series (Figure 3b-3d).

The variation trend of the kebabs is reported in Table 2 as well: it is found that both the size and periodicity of the kebabs increase with increasing pressure for the higher polymer concentration series whereas the kebabs size is less pressure dependent in the low polymer concentration series. The morphological evolution for the higher polymer concentration series is suggested as follows. Similarly, the small dots crystal at $7.79 \mathrm{MPa}$ is due to the sparse supply of P4VP in the vicinity of the CNTs under weaker $\mathrm{CO}_{2}$ antisolvent effect. Followed by a successive pressure increase within the threshold pressure value, the heterogeneous nucleation is supposed to be predominant owing to the following two reasons: (1) the viscosity and surface tension of the CXLs will decrease with increasing $\mathrm{CO}_{2}$ solubility in DMSO, which favors the P4VP adsorption on CNTs, and (2) the crystallization theory also 


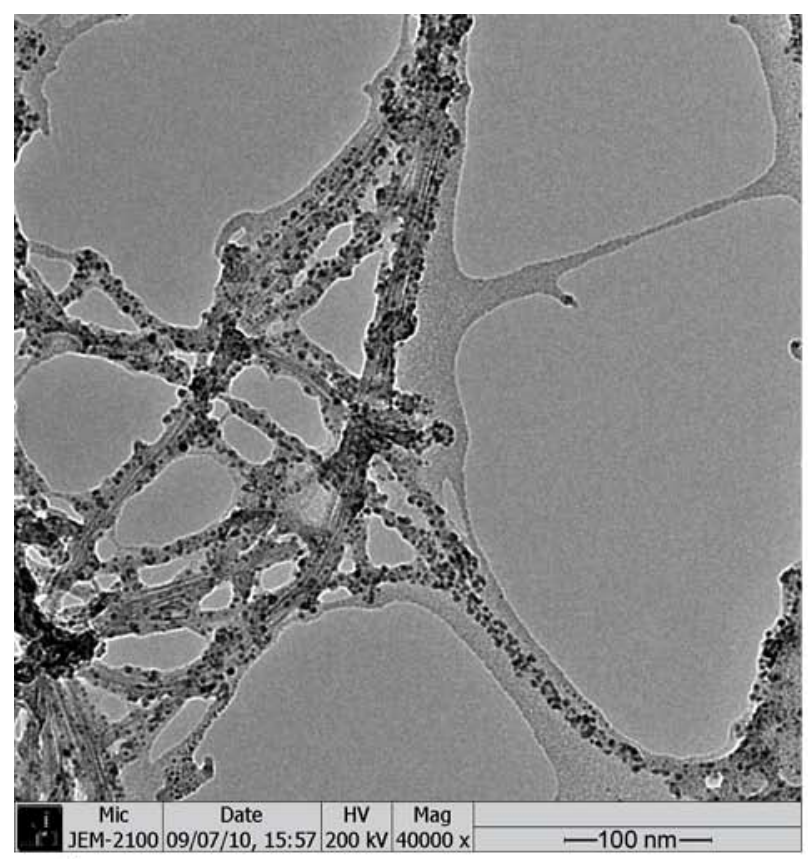

a)

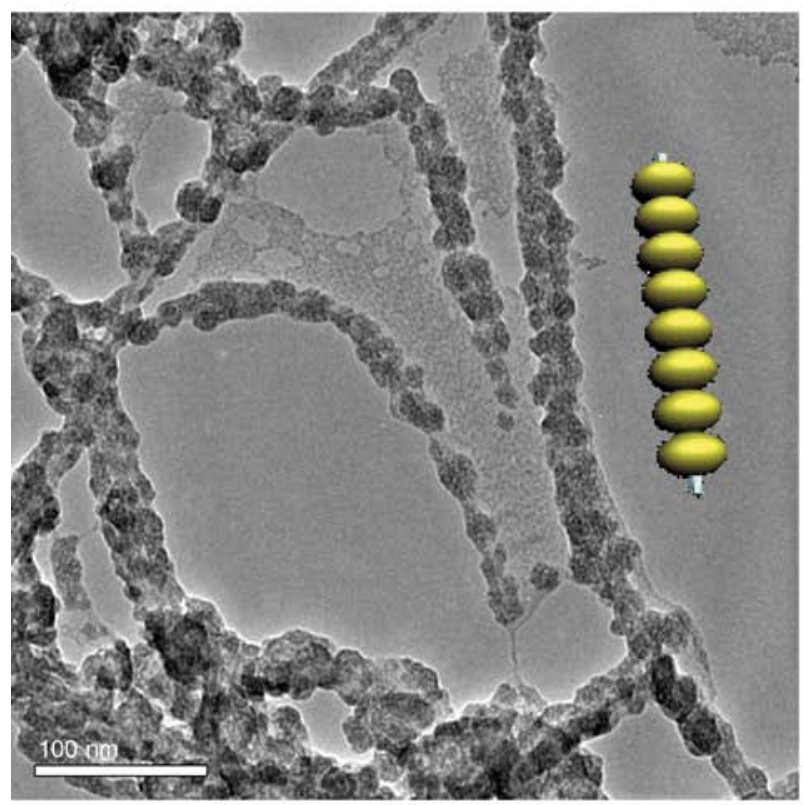

c)

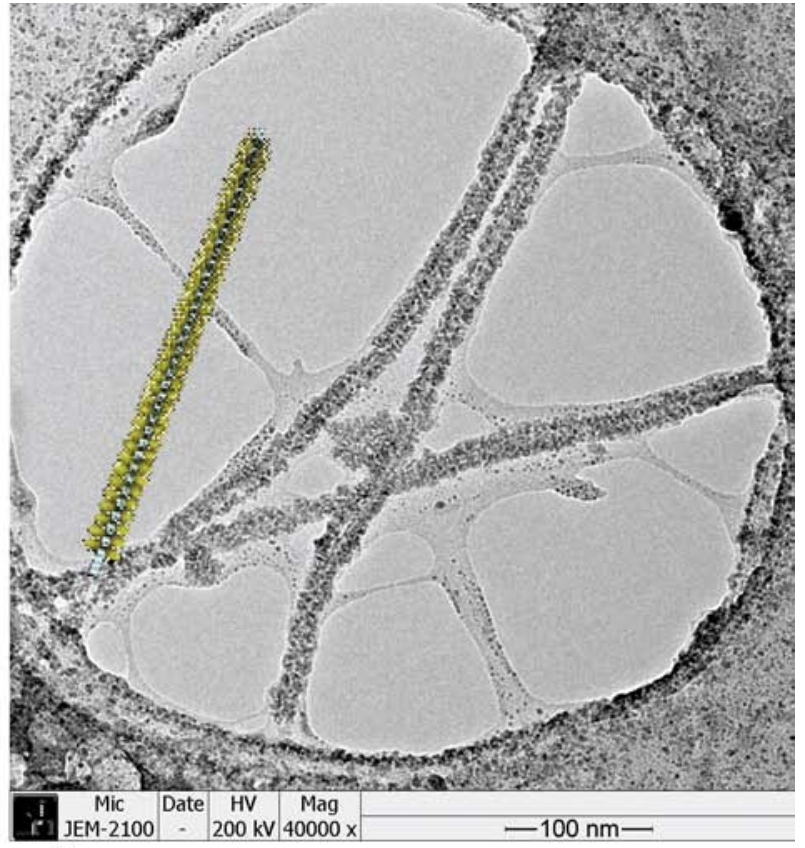

b)

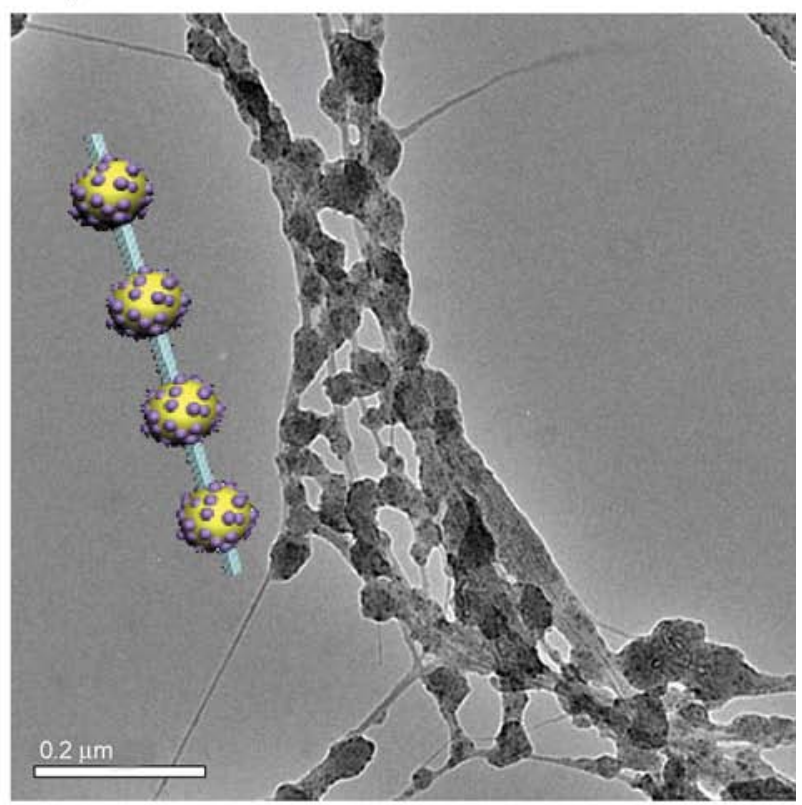

d)

Figure 3. TEM images of $\mathrm{P} 4 \mathrm{VP}_{15470} / \mathrm{SWCNTs}$ composites obtained in $\mathrm{CO}_{2}$-expanded DMSO (338.2 K/varied pressure for $3 \mathrm{~h}$ ), with $0.010 \mathrm{wt} \% \mathrm{P}_{4} \mathrm{VP}_{15470}$ and $0.002 \mathrm{wt} \%$ SWCNTs in DMSO: (a) $7.79 \mathrm{MPa}\left(x_{\mathrm{CO}_{2}}=0.399\right)$; (b) $8.30 \mathrm{MPa}$ $\left(x_{\mathrm{CO}_{2}}=0.425\right)$; (c) $8.63 \mathrm{MPa}\left(x_{\mathrm{CO}_{2}}=0.442\right)$; (d) $10.53 \mathrm{MPa}\left(x_{\mathrm{CO}_{2}}=0.543\right)$. There are schematic representations of the $\mathrm{P}_{4} \mathrm{VP}_{15470} / \mathrm{SWCNTs}$ NHSK-like structure on image (b), (c) and (d), respectively.

supports the heterogeneous nucleation. Therefore, the preferential heterogeneous nucleation would promote the growth of the kebabs with more P4VP accumulated on CNTs surface due to pressure increase, which leads to a lateral size enhancement of kebabs with increasing pressure from $8.3 \mathrm{MPa}$ (brush diameter $25 \mathrm{~nm}$, Figure 3b) to $8.63 \mathrm{MPa}$ (lateral size 20-40 nm, Figure 3c). However, if the pressure is above the threshold pressure, the com- petitive consumption of P4VP by homogeneous nucleation will dramatically slow down the heterogeneous lamellae thickening process so that less heteronucleus crystals are thickened, leading to a widely-spaced dotted kebabs conformation in the nanocomposites as shown in Figure $3 \mathrm{~d}(10.53 \mathrm{MPa}$, periodicity $50-80 \mathrm{~nm})$. A similar phenomenon is also found in the low polymer concentration series in Figure 2d-2e. Moreover, the TEM image reveals 
some difference between the 2D NHSK reflected in Figure $2 \mathrm{c}$ and the 2D NHSK in Figure $3 \mathrm{c}$. The rounded kebabs in Figure $3 \mathrm{c}$ are shorter in length and their aspect ratio is smaller as opposite to the kebabs in Figure 2c, which might be due to a much larger P4VP concentration and more heterogeneous numbers on CNTs surface in the higher polymer concentration series during the heterogeneous crystal growth process. In short, there exists notable pressure dependence of morphological evolution in $\mathrm{P}_{4} \mathrm{VP}_{15470} / \mathrm{SWCNTs}$ composites at the selective conditions. Moreover, both the P4VP decoration style and the degree on SWCNTs are more sensitive to pressure tuning for the higher $\mathrm{P}_{4} \mathrm{VP}_{15470}$ concentration series.

\subsection{Effect of molecular weight of P4VP on the modification of SWCNTs}

To reveal the MW-P4VP effect on the CNTs modification, $\mathrm{P} \mathrm{VP}_{9615}$ and $\mathrm{P} \mathrm{VP}_{15470}$ are selected, respectively. The P4VP $(0.006 \mathrm{wt} \%)$ and SWCNTs $(0.002 \mathrm{wt} \%)$ in DMSO were treated at $338.2 \mathrm{~K}$ and $9.30 \mathrm{MPa}\left(x_{\mathrm{CO}_{2}}=0.476\right)$ for $3 \mathrm{~h}$. Contrast experiments are conducted at a higher pressure around $11.30 \mathrm{MPa}\left(x_{\mathrm{CO}_{2}}=0.586\right)$. The corresponding TEM images are shown in Figure 4. It is seen that the conformation of $\mathrm{P}_{4} \mathrm{VP}_{9615}$ on SWCNTs are dots crystal with average diameter around 5-10 $\mathrm{nm}$ at 9.30 $\mathrm{MPa}$ (Figure 4a), and evenly distributed smaller dots crystal with diameter about $5 \mathrm{~nm}$ at $11.30 \mathrm{MPa}$ (Figure 4b).

In contrast, the $\mathrm{P} 4 \mathrm{VP}_{15470} / \mathrm{SWCNTs}$ composites are NHSK-like structure with compact kebabs at 9.29 MPa (Figure 2b), and less compact kebabs at

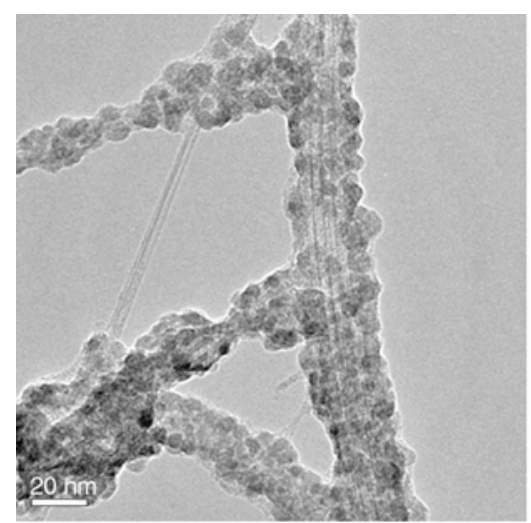

a)
11.32 $\mathrm{MPa}$ (Figure 2c). Herein, even if the pressure increase from 9.30 to $11.30 \mathrm{MPa}$, the conformations of $\mathrm{P} \mathrm{VP}_{9615}$ on SWCNTs always adopt a dot-like pattern. It might be that the solubility of $\mathrm{P} \mathrm{VP}_{9615}$ in DMSO is better than that of $\mathrm{P} \mathrm{VP}_{15470}$ at the same conditions, there is limited amount of $\mathrm{P}_{4} \mathrm{VP}_{9615}$ precipitated out disregarding the increase in CXLs pressure. Therefore, the dot-like crystals of $\mathrm{P}_{4} \mathrm{VP}_{9615}$ are formed on SWCNTs owing to the insufficient supply of P4VP on CNTs surface. However, the size of dots crystal in $\mathrm{P}_{4} \mathrm{VP}_{9615} / \mathrm{SWCNTs}$ becomes smaller with increasing pressure. Smaller size of $\mathrm{P} \mathrm{VP}_{9615}$ crystal at $11.30 \mathrm{MPa}$ means the growth of the heteronucleus P4VP dots is not rapid enough as opposed to that of the nearby homogeneous nucleation of P4VP when pressure is higher than the threshold pressure. Provided that the polymer concentration is the same, the modification results show analogous molecular weight tuning of structural transition from dots in $\mathrm{P} \mathrm{VP}_{9615} / \mathrm{SWCNTs}$ to kebabs in $\mathrm{P} \mathrm{VP}_{15470} / \mathrm{SWCNTs}$ at the selected pressures. And it seems that the CXLs method offers better morphology control over the $\mathrm{P} \mathrm{VP}_{15470} /$ SWCNTs systems. To summarize, $\mathrm{P} \mathrm{VP}_{9615}$ forms dot-like crystals on SWCNTs while P4VP 15470 forms kebab-like crystals instead at the same polymer concentration and CXLs pressure, which suggests that the P4VP crystal conformation on CNTs surface could be also regulated by the variation in the molecular weight of P4VP. It is found that only polymers with specific molecular weight (e.g. $\mathrm{P}_{4} \mathrm{VP}_{15470}$ ) can form the NHSK structure on SWCNTs.

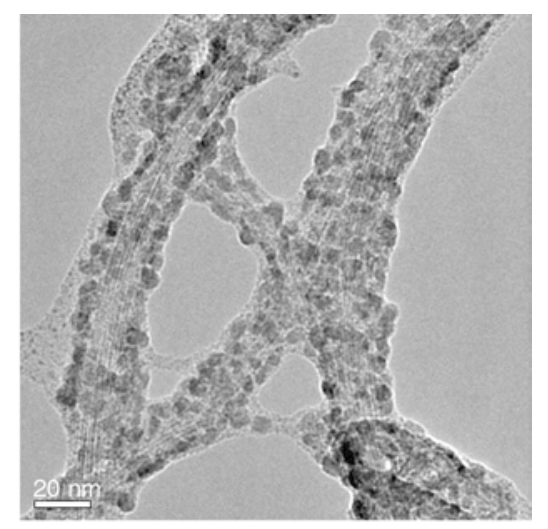

b)

Figure 4. TEM images of $\mathrm{P} 4 \mathrm{VP}_{9615} / \mathrm{SWCNTs}$ composites obtained in $\mathrm{CO}_{2}$-expanded DMSO (338.2 K/varied pressure for $3 \mathrm{~h}$ ), with $0.006 \mathrm{wt} \% \mathrm{P} 4 \mathrm{VP}_{9615}$ and $0.002 \mathrm{wt} \%$ SWCNTs in DMSO: (a) $9.30 \mathrm{MPa}\left(x_{\mathrm{CO}_{2}}=0.476\right)$; (b) $11.30 \mathrm{MPa}$ $\left(x_{\mathrm{CO}_{2}}=0.586\right)$. 


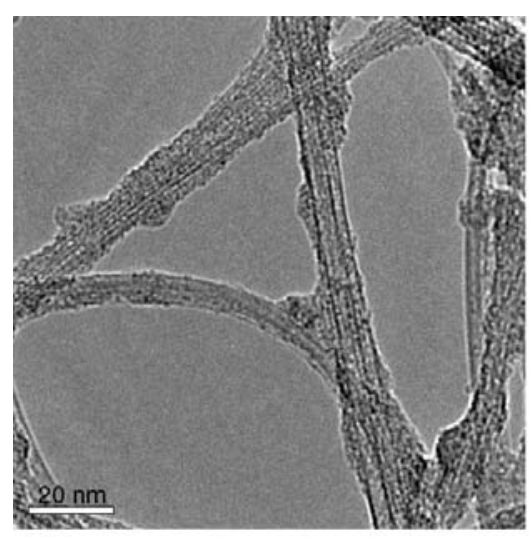

a)

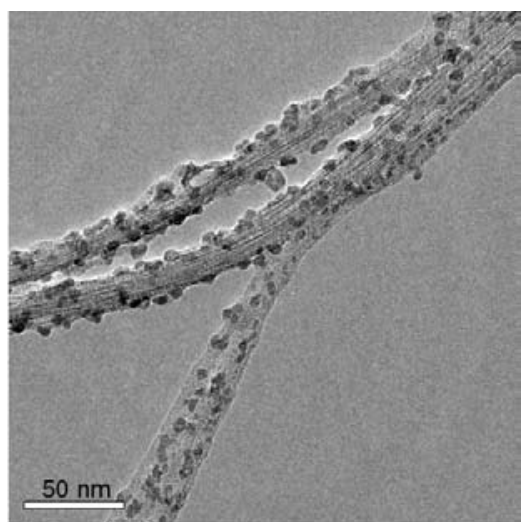

b)

Figure 5. TEM images of P4VP-decorated SWCNTs obtained in DMSO at $338.2 \mathrm{~K}$ for $3 \mathrm{~h}$. (a) $0.006 \mathrm{wt} \% \mathrm{P} 4 \mathrm{VP}_{9615}$ and $0.002 \mathrm{wt} \%$ SWCNT (b) $0.010 \mathrm{wt} \% \mathrm{P}_{4} \mathrm{VP}_{15470}$ and $0.002 \mathrm{wt} \%$ SWCNT.

\subsection{TEM image of $\mathrm{P}^{4} \mathrm{VP}_{9615} / \mathrm{SWCNTs}$ and $\mathbf{P} V_{V_{15470}}$ / SWCNTs composites by conventional method without $\mathrm{CO}_{2}$}

The TEM images of $\mathrm{P} 4 \mathrm{VP}_{9615}$ and $\mathrm{P} \mathrm{VP}_{15470}$ decorated SWCNTs under the same experimental conditions free of $\mathrm{CO}_{2}$ are shown in Figure 5, respectively. From Figure 5, the wrapping pattern of P4VP 9615 on SWCNTs (in DMSO without $\mathrm{CO}_{2}$ ) is found to be a sparsely coating structure, instead, evenly distributed dots crystal of $\mathrm{P} \mathrm{VP}_{15470}$ is formed on SWCNTs surface. In contrast, the pressure dependent SWCNTs microstruture observed in $\mathrm{P} \mathrm{VP}_{15470} / \mathrm{SWCNTs}$ composites (Figure 2 and Figure 3) or $\mathrm{P} \mathrm{VP}_{9615} / \mathrm{SWCNTs}$ composites (Figure 4) by CXLs method suggests that nanotube microstructure in composites can be tailored as a function of CXLs pressure. Therefore, it is undoubtedly that the CXLs method offers superior control of the P4VP crystallization patterns on SWCNTs in contrast with the conventional way.

\section{Conclusions}

In summary, CXLs method is a facile process for preparing P4VP/SWCNT composites with controlled polymer-crystal morphology. The MW- P4VP as well as the pressure in CXLs are the effective approaches to control the polymer crystallization structures on SWCNTs, and the crystallization behavior of $\mathrm{P}_{4} \mathrm{VP}_{15470}$ is dramatically affected by CXLs pressure. A threshold pressure around $10.00 \mathrm{MPa}\left(x_{\mathrm{CO}_{2}}=0.514\right)$ is assumed to exist in our CXLs system, and the amount and number of heterogeneous polymer nucleation is supposed to be predominating over the homogeneous one below the threshold pressure, whereas the homogeneous nucleation of polymer is advantageous or preferential above the threshold pressure. The combination of desired pressure of $\mathrm{CO}_{2}$ and MW-P4VP helps to modify the SWCNTs in a controllable way at suitable polymer concentration and temperature, which could serve as a model process to achieve ideal polymer crystals for structural and morphological study. Moreover, the P4VP crystal formation mechanism is different for SWCNTs and MWCNTs modification. The NHSK conformation of the $\mathrm{P} \mathrm{VP}_{15470} / \mathrm{SWCNTs}$ in this work is attributed to 'size-dependent soft epitaxy growth' mechanism because geometric confinement of the small diameter SWCNTs (OD 1-2 nm) is the predominant factor, which dictates the polymer chain orientation in the kebabs. Instead, 'normal epitaxy growth' mechanism was supposed to play a major role, therefore P4VP helical wrapping crystals were observed on MWCNTs (OD 25-30 nm) using the CXLs method at the same polymer concentration and comparable pressures [22]. In other words, the diameter of the CNTs also plays a critical role in the morphology control of P4VP crystals on CNTs surface. This work offers an environmentally benign polymer wrapping approach in CXLs to noncovalent engineering of SWCNTs surfaces that leads to an enhancement of solubility or dispersibility of CNTs and enables superior control of the relative placement of functionalities on the CNT surface. We believe that the pressure tuning of the unique nanoscale architecture of P4VP/SWCNT composites in CXLs could find a variety of applications ranging from nanoelectronics, sensing to catalyst supports. 


\section{Acknowledgements}

This work was supported by funds from the National Natural Science Foundation of China (No.21073167), and the Scientific Research Foundation for the Returned Overseas Chinese Scholars, State Education Ministry of China (No.2008890).

\section{References}

[1] Moniruzzaman M., Winey K. I.: Polymer nanocomposites containing carbon nanotubes. Macromolecules, 39, 5194-5205 (2006).

DOI: $10.1021 / \mathrm{ma} 060733 \mathrm{p}$

[2] Winey K. I., Vaia R. A.: Polymer nanocomposites. Materials Research Society Bulletin, 32, 314-319 (2007).

[3] Li L., Li B., Hood M. A., Li C. Y.: Carbon nanotube induced polymer crystallization: The formation of nanohybrid shish-kebabs. Polymer, 50, 953-965 (2009). DOI: $10.1016 /$ j.polymer.2008.12.031

[4] Zhang Z. W., Xu Q., Chen Z. M., Yue J.: Nanohybrid shish-kebabs: Supercritical $\mathrm{CO}_{2}$-induced PE epitaxy on carbon nanotubes. Macromolecules, 41, 2868-2873 (2008).

DOI: $10.1021 / \mathrm{ma} 702739 \mathrm{n}$

[5] Wen F., Zhang W., Wei G., Wang Y., Zhang J., Zhang M., Shi L.: Synthesis of noble metal nanoparticles embedded in the shell layer of core-shell poly(styreneco-4-vinylpyridine) micospheres and their application in catalysis. Chemistry of Materials, 20, 2144-2150 (2008).

DOI: $10.1021 / \mathrm{cm} 703378 \mathrm{c}$

[6] Yarapathi R. V., Kurva S., Tammishetti S.: Synthesis of 3,4-dihydropyrimidin-2(1H)ones using reusable poly(4-vinylpyridine-co-divinylbenzene)-Cu(II)complex. Catalysis Communications, 5, 511-513 (2004). DOI: $10.1016 /$ j.catcom.2004.06.007

[7] Friedrich H. B., Singh N.: The very efficient oxidation of alcohols by poly(4-vinylpyridine)-supported sodium ruthenate. Tetrahedron Letters, 41, 3971-3974 (2000). DOI: 10.1016/S0040-4039(00)00531-1

[8] Jackson J. A., Newsham M. D., Worsham C., Nocera D. G.: Efficient singlet oxygen generation from polymers derivatized with hexanuclear molybdenum clusters. Chemistry of Materials, 8, 558-564 (1996). DOI: $10.1021 / \mathrm{cm} 950443 \mathrm{f}$

[9] Chen Y. S., Li Y., Yang M. J.: A fast response resistive thin film humidity sensor based on poly(4-vinylpyridine) and poly(glycidyl methacrylate). Journal of Applied Polymer Science, 105, 3470-3475 (2007). DOI: 10.1002/app.26401

[10] Tiller J. C., Lee S. B., Lewis K., Klibanov A. M.: Polymer surfaces derivatized with poly(vinyl-N-hexylpyridinium) kill airborne and waterborne bacteria. Biotechnology and Bioengineering, 79, 465-471 (2002). DOI: 10.1002/bit.10299
[11] Minko S., Kiriy A., Gorodyska G., Stamm M.: Single flexible hydrophobic polyelectrolyte molecules adsorbed on solid substrate: Transition between a stretched chain, necklace-like conformation and a globule. Journal of the American Chemical Society, 124, 3218-3219 (2002).

DOI: $10.1021 / j a 017767 \mathrm{r}$

[12] Kiriy A., Gorodyska G., Minko S., Jaeger W., Štěpánek P., Stamm M.: Cascade of coil-globule conformational transitions of single flexible polyelectrolyte molecules in poor solvent. Journal of the American Chemical Society, 124, 13454-13462 (2002). DOI: $10.1021 / \mathrm{ja0261168}$

[13] Li Y., Yang M. J., She Y.: Humidity sensitive properties of crosslinked and quaternized poly(4-vinylpyridine-co-butyl methacrylate). Sensors and Actuators B: Chemical, 107, 252-257 (2005). DOI: $10.1016 /$ j.snb.2004.10.008

[14] Aydogdu Y., Erol I., Yakuphanoğlu F., Aydogdu A., Ahmedzade M.: Electrical conductivity and optical properties of copolymers based on 4-vinylpyridine and tetralincyclobutylhydroxyethylmethacrylate. Synthetic Metals, 139, 327-334 (2003). DOI: 10.1016/S0379-6779(03)00183-8

[15] Anand M., You S-S., Hurst K. M., Saunders S. R., Kitchens C. L., Ashurst W. R., Roberts C. B.: Thermodynamic analysis of nanoparticle size selective fractionation using gas-expanded liquids. Industrial and Engineering Chemistry Research, 47, 553-559 (2008). DOI: 10.1021/ie070981p

[16] Dehghani F., Foster N. R.: Dense gas anti-solvent processes for pharmaceutical formulation. Current Opinion in Solid State and Materials Science, 7, 363369 (2003). DOI: $10.1016 /$ j.cossms.2003.11.001

[17] Olesik S. V.: Physicochemical properties of enhancedfluidity liquid solvents. Journal of Chromatography A, 1037, 405-410 (2004).

DOI: $10.1016 /$ j.chroma.2004.04.001

[18] Eckert C. A., Liotta C. L., Bush D., Brown J. S., Hallett J. P.: Sustainable reactions in tunable solvents. Journal of Physical Chemistry B, 108, 18108-18118 (2004).

DOI: $10.1021 / \mathrm{jp} 0487612$

[19] Wei M., Musie G. T., Busch D. H., Subramaniam B.: Autoxidation of 2,6-di-tert-butylphenol with cobalt Schiff base catalysts by oxygen in $\mathrm{CO}_{2}$-expanded liquids. Green Chemistry, 6, 387-393 (2004). DOI: $10.1039 / \mathrm{B} 310523 \mathrm{G}$

[20] Lyon C. J., Sarsani V. R., Subramaniam B.: 1-Butene + isobutane reactions on solid acid catalysts in dense $\mathrm{CO}_{2}$-based reaction media: Experiments and modeling. Industrial and Engineering Chemistry Research, 43, 4809-4814 (2004). DOI: $10.1021 / \mathrm{ie} 0498145$ 
[21] Jessop P. G., Subramaniam B.: Gas-expanded liquids. Chemical Reviews, 107, 2666-2694 (2007).

DOI: $10.1021 / \mathrm{cr} 0401990$

[22] Liu Y., Wei Y. N., Qin R. H., Zhang L. Y., Yang L. T., Li H. P., Li X. J.: Crystalline polymer decoration on multiwalled carbon nanotubes: MWCNT-induced P4VP periodic crystallization in $\mathrm{CO}_{2}$-expanded liquids. Express Polymer Letters, 5, 60-72 (2011).

DOI: 10.3144/expresspolymlett.2011.7

[23] Fischer A., Brembilla A., Lochon P.: Nitroxide-mediated radical polymerization of 4-vinylpyridine: Study of the pseudo-living character of the reaction and influence of temperature and nitroxide concentration. Macromolecules, 32, 6069-6072 (1999).

DOI: $10.1021 / \mathrm{ma990116j}$

[24] Chalari I., Pispas S., Hadjichristidis N.: Controlled free-radical polymerization of 2-vinylpyridine in the presence of nitroxides. Journal of Polymer Science Part A: Polymer Chemistry, 39, 2889-2895 (2001).

DOI: $10.1002 /$ pola.1268
[25] Chiu H-Y., Jung R-F., Lee M-J., Lin H-M.: Vapor-liquid phase equilibrium behavior of mixtures containing supercritical carbon dioxide near critical region. The Journal of Supercritical Fluids, 44, 273-278 (2008). DOI: 10.1016/j.supflu.2007.09.026

[26] Zhang F., Zhang H., Zhang Z., Chen Z., Xu Q.: Modification of carbon nanotubes: Water-soluble polymers nanocrystal wrapping to periodic patterning with assistance of supercritical $\mathrm{CO}_{2}$. Macromolecules, 41, 4519-4523 (2008). DOI: $10.1021 / \mathrm{ma} 800514 \mathrm{a}$

[27] Zhang L., Tao T., Li C.: Formation of polymer/carbon nanotubes nano-hybrid shish-kebab via non-isothermal crystallization. Polymer, 50, 3835-3840 (2009). DOI: $10.1016 /$ j.polymer.2009.05.051

[28] Flory P. J.: Principles of polymer chemistry. Cornell University Press, Ithaca (1953). 